\title{
European Bifurcation Club Perspectives on Two-stent Techniques - An Interview
}

\author{
An Expert Interview with Dr Francesco Burzotta
}

Institute of Cardiology, Catholic University of the Sacred Heart, Rome, Italy

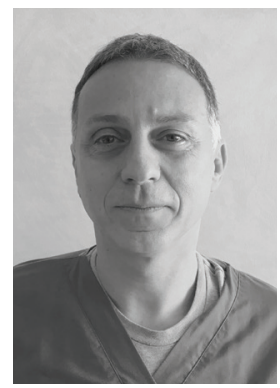

\section{Francesco Burzotta}

Dr Francesco Burzotta is an interventional cardiologist at the Institute of Cardiology, Catholic University of the Sacred Heart, Rome, Italy. After training in medicine and specialising in cardiology at the Catholic University of the Sacred Heart, Dr Burzotta has held the position of interventional cardiologist at this institution since 2003. He has published over 300 papers, with a specialist focus on interventional procedures and is a board member of the European Bifurcation Club.

\section{Keywords}

Two-stent, percutaneous coronary intervention, bifurcated lesions, techniques

Disclosures: Francesco Burzotta has received speaker's fees from Abiomed, Medtronic and Abbott. Acknowledgement: Medical writing assistance was provided by Dr CG Griffin of Griffin Scientific Ltd on behalf of Touch Medical Media and was supported by Touch Medical Media.

Review Process: This is an expert interview and, as such, has not undergone the journal's standard peer review process.

Compliance with Ethics: This article is an interview and did not involve any studies with human or animal subjects performed by any of the authors.

Authorship: The named author meets the International Committee of Medical Journal Editors (ICMJE) criteria for authorship of this manuscript, takes responsibility for the integrity of the work as a whole, and has given final approval for the version to be published.

Received: 25 July 2019

Published Online: 18 September 2019 Citation: Heart International. 2019;13(1):9-10

Corresponding Author: Dr Francesco Burzotta, MD, $\mathrm{PhD}$, Institute of Cardiology, Fondazione Policlinico Universitario A. Gemelli IRCCS, Universita Cattolica del Sacro Cuore, L.go Gemelli 1, 00141 Rome, Italy

E·francescoburzotta@gmail.com

Support: No funding was received for

the publication of this article.
$\mathrm{T}$ he treatment of coronary bifurcation lesions remains a key clinical challenge in interventional cardiology. Short-term trials have shown benefits with a provisional, one-stent approach, and these findings have been supported by longer-term data when compared with intentional, up-front two-stent approaches. ${ }^{1,2}$ However, these broad observations do not translate to practical guidance for physicians who may need to consider intentional two-stent approaches for individuals with complex coronary lesions. The European Bifurcation Club (EBC) has published its fourteenth consensus, providing practical guidance on percutaneous coronary intervention for obstructive bifurcation lesions. ${ }^{3}$ It aims to provide support to physicians who are managing bifurcation disease.

\section{Q. What are the key messages of the European Bifurcation Club 2019 Consensus Statement on Two-stent Techniques?}

The EBC is a group of scientists and clinicians drawing on a range of multidisciplinary skills that are focussed on improving treatment outcomes in patients with coronary bifurcation disease. Periodically, this group reviews emerging evidence along with clinical experience to help refine understanding and contribute to practical guidance. In seeking to optimise bifurcation treatment, the group examined the role of two-stent techniques.

Two-stent bifurcation treatment is a complex procedure and the technique can vary between institutions and individual physicians. It has been considered by the EBC that the way the procedure is performed can influence treatment effectiveness and they sought to examine current best practice.

Due to the complex nature of all bifurcation double stenting techniques, it was evident that a clear plan for both immediate and long-term management is essential. Elective two-stent approaches may benefit patients with complex lesions and the need for a two-stent technique may become apparent during the steps of bifurcation stenting procedures started with a single stent implantation. In terms of optimising the two-stent procedure, the EBC notes that final kissing-balloon inflation improves the distribution of the stent's struts in the bifurcation area. ${ }^{3}$

The EBC percutaneous coronary intervention consensus provides advice across currently available techniques and aims to support physicians in their preferred approaches to treatment. It is hoped that these insights and practical suggestions will help to improve outcomes in the challenging field of treating bifurcation disease. 


\section{Q. How should physicians incorporate this consensus into their decision making when considering one- and two-stent treatment approaches?}

The most important message is to understand the scope, complexity, steps involved and time required for successful two-stent techniques. single-stent approaches are simpler to perform and the EBC notes that where simple lesions need to be treated, the single-stent technique is the most appropriate.

When complex lesions need to be managed, physician experience with techniques is an important consideration. The EBC notes that a two-stent approach can be a valuable approach in selected patients with complex coronary bifurcation lesions. ${ }^{3}$ ]

Behan MW, Holm NR, de Belder AJ, et al. Coronary bifurcation lesions treated with simple or complex stenting: 5 -year survival from patient-level pooled analysis of the Nordic Bifurcation Study and the British Bifurcation Coronary Study. Eur Heart J. 2016;37:1923-8.

2. Ford TJ, McCartney P, Corcoran D, et al. Single- versus 2-stent strategies for coronary bifurcation lesions: a systematic review and meta-analysis of randomized trials with long-term follow-up. $J$ Am Heart Assoc. 2018; 7:pii:e008730.

3. Banning AP, Flensted Lassen J, Burzotta F, et al. Percutaneous coronary intervention for obstructive bifurcation lesions: the 14th consensus document from the European Bifurcation Club. EBC 2019 Consensus on two-stent treatment of bifurcation. Eurolntervention. 2019;15:90-8. 